\title{
A Modified Lanczos Algorithm for the Computation of Transient Electromagnetic Wavefields
}

\author{
Rob F. Remis and Peter M. van den Berg
}

\begin{abstract}
A new method for computing transient electromagnetic wavefields in inhomogeneous and lossy media is presented. The method utilizes a modified Lanczos scheme, where a socalled reduced model is constructed. A discretization of the time variable is then superfluous. This reduced model represents the transient electromagnetic wavefield on a certain bounded interval in time. Some theoretical aspects of the method are highlighted and numerical results showing the performance of the method for two-dimensional (2-D) configurations are given. Also, comparisons between this Lanczos method and the finitedifference time-domain (FDTD) method are made.
\end{abstract}

Index Terms - Lanczos algorithm, reduced models, transient electromagnetic wavefields.

\section{INTRODUCTION}

A STANDARD method for computing transient electromagnetic wavefields is the finite-difference time-domain (FDTD) method [1]. In this method, Maxwell's equations are discretized in space and time, employing central finite differences for the derivatives with respect to the space and time variables. A disadvantage of the FDTD method is that the time step is limited by the Courant-Friedrichs-Lewy stability condition [1]. Another disadvantage is the fact that if one is interested in the frequency response from the timedomain results, the time step must be chosen very small; hence, increasing the computational work that is involved. In case one is dealing with diffusive electromagnetic fields (neglecting the displacement currents), explicit time-stepping methods are even more time consuming, since for these fields, the Courant-Friedrichs-Lewy stability condition puts stricter limitation on the time step compared to the wavefield case. To be more precise, if $\Delta x$ denotes the minimum spatial increment and if $\Delta t$ denotes the time step, then the number of time steps to get a solution at time $t$ is proportional to $t / \Delta x$ for wavefield problems and $t /(\Delta x)^{2}$ for diffusion problems.

Recently, Druskin and Knizhnerman [2] have presented a much more efficient approach for computing transient diffusive electromagnetic fields in inhomogeneous media using the method of Lanczos-the spectral Lanczos decomposition method (SLDM). Their approach is based on a second-order differential equation for either the electric- or magnetic-field

Manuscript received November 18, 1996; revised June 5, 1997. This work was supported by the Technology Foundation (STW).

The authors are with the Laboratory of Electromagnetic Research, Faculty of Electrical Engineering, Centre for Technical Geoscience, Delft University of Technology, The Netherlands (e-mail: r.f.remis@ctg.tudelft.nl; p.m.vdberg@et.tudelft.nl).

Publisher Item Identifier S 0018-9480(97)08247-1. strength. One of the characteristics of the method is that it is not an explicit time-stepping method.

The present method of this paper differs from the approach of Druskin and Knizhnerman in the following respects. Firstly, we are dealing with the wave (hyperbolic-type) equations rather than the diffusive (parabolic-type) equations. Secondly, we consider Maxwell's equations as a system of first-order partial differential equations rather than two separate equations of second order. In our method, a modified Lanczos algorithm is developed in such a way that the time variable does not need to be discretized, i.e., our method is not an explicit timestepping method. Some special properties of the method are analyzed theoretically and also an error analysis is presented, from which the number of Lanczos iterations required within a finite time interval of observation follows. For convenience, numerical results will be shown for various two-dimensional (2-D) configurations only and comparisons between the results obtained via the FDTD method and our method will be made.

\section{BASIC EQUATIONS}

To specify position in a domain in which an inhomogeneous, anisotropic, and lossy medium is present, we employ the vector $x$ with Cartesian coordinates $x_{1}, x_{2}$, and $x_{3}$. Further, $\partial_{1}, \partial_{2}$, and $\partial_{3}$ denote differentiation with respect to $x_{1}, x_{2}$, and $x_{3}$, respectively, while $\partial_{t}$ denotes differentiation with respect to the time coordinate $t$.

The point-wise behavior of the electromagnetic field is described by Maxwell's equations written here in the form

$$
\left(\mathcal{D}+\mathcal{M}_{1}+\mathcal{M}_{2} \partial_{t}\right) \mathcal{F}=\mathcal{Q}^{\prime}
$$

where $\mathcal{D}$ is a symmetric spatial differential operator matrix given by

$$
\mathcal{D}=\left(\begin{array}{rrrrrr}
0 & 0 & 0 & 0 & \partial_{3} & -\partial_{2} \\
0 & 0 & 0 & -\partial_{3} & 0 & \partial_{1} \\
0 & 0 & 0 & \partial_{2} & -\partial_{1} & 0 \\
0 & -\partial_{3} & \partial_{2} & 0 & 0 & 0 \\
\partial_{3} & 0 & -\partial_{1} & 0 & 0 & 0 \\
-\partial_{2} & \partial_{1} & 0 & 0 & 0 & 0
\end{array}\right)
$$

and the time-independent matrices $\mathcal{M}_{1}$ and $\mathcal{M}_{2}$ are medium matrices given by

$$
\mathcal{M}_{1}=\left(\begin{array}{cccccc}
\sigma_{1,1} & \sigma_{1,2} & \sigma_{1,3} & 0 & 0 & 0 \\
\sigma_{2,1} & \sigma_{2,2} & \sigma_{2,3} & 0 & 0 & 0 \\
\sigma_{3,1} & \sigma_{3,2} & \sigma_{3,3} & 0 & 0 & 0 \\
0 & 0 & 0 & 0 & 0 & 0 \\
0 & 0 & 0 & 0 & 0 & 0 \\
0 & 0 & 0 & 0 & 0 & 0
\end{array}\right)
$$


and

$$
\mathcal{M}_{2}=\left(\begin{array}{cccccc}
\varepsilon_{1,1} & \varepsilon_{1,2} & \varepsilon_{1,3} & 0 & 0 & 0 \\
\varepsilon_{2,1} & \varepsilon_{2,2} & \varepsilon_{2,3} & 0 & 0 & 0 \\
\varepsilon_{3,1} & \varepsilon_{3,2} & \varepsilon_{3,3} & 0 & 0 & 0 \\
0 & 0 & 0 & \mu_{1,1} & \mu_{1,2} & \mu_{1,3} \\
0 & 0 & 0 & \mu_{2,1} & \mu_{2,2} & \mu_{2,3} \\
0 & 0 & 0 & \mu_{3,1} & \mu_{3,2} & \mu_{3,3}
\end{array}\right) .
$$

Using energy considerations it can be shown that the permittivity matrix $\varepsilon_{i, j}=\varepsilon_{i, j}(x)$ and the permeability matrix $\mu_{i, j}=\mu_{i, j}(x)$ are symmetric and positive definite. The conductivity matrix $\sigma_{i, j}=\sigma_{i, j}(x)$ is positive semidefinite and is taken to be symmetric. Hence, the medium matrix $\mathcal{M}_{1}$ is symmetric and positive semidefinite and the medium matrix $\mathcal{M}_{2}$ is symmetric and positive definite. Further, the field vector $\mathcal{F}=\mathcal{F}(x, t)$ consists of the components of the electric-field strength $\boldsymbol{E}$ and the components of the magnetic-field strength $\boldsymbol{H}$ and is given by

$$
\mathcal{F}=\left[E_{1}, E_{2}, E_{3}, H_{1}, H_{2}, H_{3}\right]^{T}
$$

while the source vector $\mathcal{Q}^{\prime}=\mathcal{Q}^{\prime}(x, t)$ is composed of the components of the external electric-current source $\boldsymbol{J}^{\mathrm{e}}$ and the components of the external magnetic-current source $\boldsymbol{K}^{\mathrm{e}}$

$$
\mathcal{Q}^{\prime}=-\left[J_{1}^{\mathrm{e}}, J_{2}^{\mathrm{e}}, J_{3}^{\mathrm{e}}, K_{1}^{\mathrm{e}}, K_{2}^{\mathrm{e}}, K_{3}^{\mathrm{e}}\right]^{T} .
$$

We also introduce the signature matrix $\delta^{-}$as

$$
\delta^{-}=\operatorname{diag}(1,1,1,-1,-1,-1)
$$

and we observe that the signature matrix $\delta^{-}$anticommutes with matrix $\mathcal{D}$, i.e.,

$$
\mathcal{D} \delta^{-}=-\delta^{-} \mathcal{D}
$$

and that it commutes with the medium matrices $\mathcal{M}_{1}$ and $\mathcal{M}_{2}$, i.e.,

$$
\mathcal{M}_{1} \delta^{-}=\delta^{-} \mathcal{M}_{1}
$$

and

$$
\mathcal{M}_{2} \delta^{-}=\delta^{-} \mathcal{M}_{2}
$$

Let the source vector be of the form $\mathcal{Q}(x, t)=w(t) \mathcal{Q}(x)$, where $w(t)$ is the source wavelet that vanishes for $t<0$ and $\mathcal{Q}$ is a time-independent vector. Then, because of causality, the field vector $\mathcal{F}$ must vanish everywhere for $t<0$. Now take the one-sided Laplace transform of (1) with respect to time. The resulting matrix equation is then given by

$$
\left(\mathcal{D}+\mathcal{M}_{1}+s \mathcal{M}_{2}\right) \hat{\mathcal{F}}(x, s)=\hat{w}(s) \mathcal{Q}(x)
$$

with $\operatorname{Re}(s)>0$. To show the notation employed, the expression for $\hat{\mathcal{F}}(x, s)$ is given by

$$
\hat{\mathcal{F}}(x, s)=\int_{t=0}^{\infty} \exp (-s t) \mathcal{F}(x, t) d t .
$$

In our further analysis, the Laplace-transform-parameter $s$ is taken to be real and positive. Then, Lerch's theorem (see Widder [3] or Henrici [4]) ensures that there is a one-toone correspondence between a causal time function and its Laplace-transform-domain counterpart, provided that the time function is continuous and is, at most, of exponential growth, as $t \rightarrow \infty$ and that equality in the definition of the Laplace transform (12) is invoked at the real set of points $\left\{s_{n}=\right.$ $\left.s_{0}+n h ; n=0,1,2, \cdots,\right\}$, where $s_{0}$ is sufficiently large and positive and $h$ is positive.

Subsequently, we discretize in space using standard spatial finite differences [5] with a Dirichlet boundary condition. We do not discuss here the errors made in the spatial discretization (for a discussion on this see [6]). Further, the impact of using absorbing-boundary conditions is not studied in this paper. In our test problems, we take the boundary of the domain of computation sufficiently far so that no reflections due to this boundary are observed in the points of observation. The discrete counterparts of $\mathcal{D}, \mathcal{M}_{1}, \mathcal{M}_{2}, \mathcal{F}$, and $\mathcal{Q}$ are given by $D, M_{1}, M_{2}, F$, and $Q$, respectively, and satisfy the algebraic matrix equation

$$
\left(D+M_{1}+s M_{2}\right) \hat{F}(s)=\hat{w}(s) Q
$$

with $s \in \mathbb{R}^{+}$. The matrices $D, M_{1}$, and $M_{2}$ are all square $N \times N$ matrices. Matrix $D$ is real and skew-symmetric, the matrices $M_{1}$ and $M_{2}$ are both symmetric, $M_{1}$ being semipositive definite and $M_{2}$ being positive definite. The discrete counterpart of matrix $\delta^{-}$is also denoted by $\delta^{-}$. In the discrete version, the relations

$$
\begin{aligned}
D \delta^{-} & =-\delta^{-} D \\
M_{1} \delta^{-} & =\delta^{-} M_{1}
\end{aligned}
$$

and

$$
M_{2} \delta^{-}=\delta^{-} M_{2}
$$

hold as well. We note that the chosen discretization of finite differences in space is not a restriction to our approach of the problem. One can choose any spatial discretization as long as (14)-(16) are satisfied.

Finally, (13) is rewritten as

$$
\hat{F}(s)=\hat{w}(s) M_{2}^{-1}(A+s E)^{-1} Q
$$

with $s \in \mathbb{R}^{+}$. Here, $E$ is the identity matrix and matrix $A$ is defined as

$$
A=\left(D+M_{1}\right) M_{2}^{-1} .
$$

It is noted that for some particular value of $s$, the solution of (17) can be obtained with e.g., a conjugate-gradient iterative scheme for nonsymmetric matrices [7]. However, since we are interested in many values of $s$ (or in the complete time-domain result) we would like to keep $s$ as a free parameter. Then, a Lanczos type of algorithm is necessary.

\section{A. Time-Domain Result}

Via inspection, the unique and causal time-domain counterpart of the vector $(A+s E)^{-1} Q$, is obtained as $\chi(t) \exp (-A t) Q$, where $\chi(t)$ denotes the Heaviside unit step function. The time-domain counterpart of the vector $\hat{F}(s)$ then follows as

$$
F(t)=w(t) * \chi(t) M_{2}^{-1} \exp (-A t) Q
$$


where $*$ denotes convolution in time. Computing the vector $\exp (-A t) Q$ by using any decomposition of matrix $A$, independent of $t$, is not feasible due to the large size of this matrix. The order of matrix $A$ ranges from $N=O\left(10^{5}\right)$ for 2-D problems to $N=O\left(10^{7}\right)$ for three-dimensional (3-D) problems. For example, in a 3-D configuration there are six equations describing the behavior of the electromagnetic field (Maxwell's equations written out in components). Using 200 sample points in each direction, the order of matrix $A$ becomes $6 \cdot 200^{3}=4.8 \cdot 10^{7}=O\left(10^{7}\right)$.

\section{A Modified Lanczos Algorithm}

Since computing the field vector $F(t)$ [as given by (19)] is not practicable, we will construct so-called reduced-model approximations to this vector. These approximations are all based on a modified Lanczos algorithm. This algorithm constructs a basis for a Krylov subspace of matrix $A$ with respect to the source vector $Q$. The information present in this subspace is then used in the reduced-model approximations for a continuum of time instances within a certain bounded interval in time. In order to apply a Lanczos algorithm for constructing the approximations, we introduce the bilinear form $\langle\cdot, \cdot\rangle_{\mathrm{b}}=\left\langle M_{2}^{-1} \delta^{-} \cdot, \cdot\right\rangle$, where $\langle\cdot, \cdot\rangle$ denotes the standard inner product of two real vectors. From (14) to (16), it then follows that matrix $A$ is symmetric with respect to this bilinear form. Using this symmetry property, we can define the following Lanczos algorithm based upon the three-term recurrence relation $A v_{i}=\beta_{i+1} v_{i+1}+\alpha_{i} v_{i}+\beta_{i} v_{i-1}$, namely,

$$
\left.\begin{array}{rl}
\beta_{1} v_{1} & =Q \\
w_{i} & =A v_{i}-\beta_{i} v_{i-1} \\
\alpha_{i} & =\left\langle v_{i}, w_{i}\right\rangle_{\mathrm{b}} \\
\beta_{+1} v_{i+1} & =w_{i}-\alpha_{i} v_{i}
\end{array}\right\}, \quad i=1,2, \cdots
$$

with $v_{0}=0$. (For more on the Lanczos algorithm see [8] and [9]). In each step, $\beta_{i}$ is determined from the condition that $\left\langle v_{i}, v_{i}\right\rangle_{\mathrm{b}}=1$ for $i \geq 1$. Implementation of this algorithm requires only two $N$-vectors of storage. Moreover, since matrix $A$ is sparse, the whole process is of order $N$. We can summarize $m$ steps of the modified Lanczos algorithm as

$$
A V_{m}=V_{m} T_{m}+\beta_{m+1} v_{m+1} e_{m}^{T}
$$

where matrix $V_{m}$ has the column partitioning $V_{m}=$ $\left(v_{1}, v_{2}, \cdots, v_{m}\right)$ and $T_{m}=\operatorname{tridiag}\left(\beta_{i}, \alpha_{i}, \beta_{i+1}\right)$ is a tridiagonal complex symmetric $m \times m$ matrix. The vector $e_{m}$ is the $m$ th column of the $m \times m$ identity matrix $E_{m}$. We are interested in situations where $m$ is much smaller than $N$, the order of the matrices occurring in (13) (see Fig. 1).

In Section IV, we show that by increasing the number of Lanczos steps, the reduced-model approximations converge to the field vector $F(t)$ on a finite time interval of observation. This is not to be confused with propagation problems in the frequency domain, where one wants to compute the square root of an operator using a Lanczos scheme [10].

\section{THE REDUCED-MODEL APPROXIMATIONS}

As a consequence of our modified Lanczos algorithm, we have the following theorem.

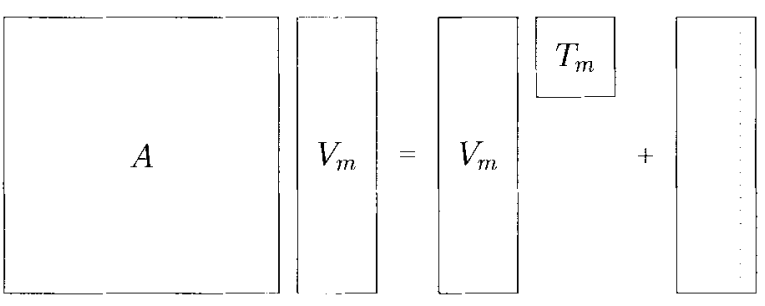

Fig. 1. A picture of (21) for $m \ll N$.

Theorem 1: Let $\beta_{1} v_{1}=Q$. After $m$ steps of the modified Lanczos algorithm we have

$$
A^{j} Q=\beta_{1} V_{m} T_{m}^{j} e_{1}, \quad j=0,1,2, \cdots, m-1 .
$$

Proof: [11]. The proof is by induction over $j$. For $j=0$, we have

$$
Q=\beta_{1} V_{m} e_{1}=\beta_{1} v_{1}
$$

and by the induction hypothesis we have that

$$
\begin{aligned}
A^{j+1} Q & =A A^{j} Q=\beta_{1} A V_{m} T_{m}^{j} e_{1} \\
& =\beta_{1}\left(V_{m} T_{m}+\beta_{m+1} v_{m+1} e_{m}^{T}\right) T_{m}^{j} e_{1} \\
& =\beta_{1} V_{m} T_{m}^{j+1} e_{1}
\end{aligned}
$$

since $e_{m}^{T} T_{m}^{j} e_{1}=0$ for $j \leq m-2$.

Note that the proof of the theorem does not rely on the orthogonality property of the Lanczos vectors $v_{i}$ with respect to our bilinear form. Equation (21) and $\beta_{1} v_{1}=Q$ were the only two equations used in the proof of the theorem.

Now consider the computation of the vector $(A+s E)^{-1} Q$, with $s>s_{0}$ and $s_{0}$ defined by

$$
s_{0}=\max \left\{\|A\|,\left\|T_{m}\right\|\right\}
$$

where $\|\cdot\|$ denotes the matrix two-norm. This vector can be written as

$$
(A+s E)^{-1} Q=\frac{1}{s} \sum_{k=0}^{m-1}\left(-\frac{1}{s} A\right)^{k} Q+\frac{1}{s} \sum_{k=m}^{\infty}\left(-\frac{1}{s} A\right)^{k} Q
$$

with $s>s_{0}$. With the aid of the last theorem, this can be written as

$$
\begin{aligned}
(A & +s E)^{-1} Q \\
& =\beta_{1} V_{m} \frac{1}{s} \sum_{k=0}^{m-1}\left(-\frac{1}{s} T_{m}\right)^{k} e_{1}+\frac{1}{s} \sum_{k=m}^{\infty}\left(-\frac{1}{s} A\right)^{k} Q \\
& =\beta_{1} V_{m}\left(T_{m}+s E_{m}\right)^{-1} e_{1}+\hat{R}_{m}(s)
\end{aligned}
$$

for $s>s_{0}$, and where the vector $\hat{R}_{m}(s)$ is given by

$$
\begin{aligned}
\hat{R}_{m}(s)= & \frac{1}{s} \sum_{k=m}^{\infty}\left[\left(-\frac{1}{s} A\right)^{k} Q-\beta_{1} V_{m}\left(-\frac{1}{s} T_{m}\right)^{k} e_{1}\right] \\
= & (-A)^{m} \frac{1}{s^{m}}(A+s E)^{-1} Q \\
& -\beta_{1} V_{m}\left(-T_{m}\right)^{m} \frac{1}{s^{m}}\left(T_{m}+s E_{m}\right)^{-1} e_{1} .
\end{aligned}
$$

The infinite series in (26)-(28) are convergent since $s>$ $s_{0}$. Then, Lerch's theorem states that there is a one-to-one 
correspondence with a causal time function. Via inspection, the causal time-domain counterpart of (27), is obtained as

$$
\chi(t) \exp (-A t) Q=\chi(t) \beta_{1} V_{m} \exp \left(-T_{m} t\right) e_{1}+R_{m}(t)
$$

where the vector $R_{m}(t)$ is given by

$$
\begin{aligned}
R_{m}(t) & \\
=\chi(t)\{ & \frac{(-A)^{m}}{(m-1) !} \int_{\tau=0}^{t} \exp [-A(t-\tau)] \tau^{m-1} d \tau Q \\
& \left.-\beta_{1} V_{m} \frac{\left(-T_{m}\right)^{m}}{(m-1) !} \int_{\tau=0}^{t} \exp \left[-T_{m}(t-\tau)\right] \tau^{m-1} d \tau e_{1}\right\} .
\end{aligned}
$$

Substitution of (29) into (19) leads to

$$
F(t)=F_{m}(t)+w(t) * M_{2}^{-1} R_{m}(t)
$$

where we have defined the reduced model $F_{m}(t)$ as

$$
F_{m}(t)=w(t) * \chi(t) \beta_{1} M_{2}^{-1} V_{m} \exp \left(-T_{m} t\right) e_{1}
$$

In Appendix B, it is shown that this reduced model is realvalued. The reduced model $F_{m}(t)$ is now taken as an approximation to the vector $F(t)$ on a certain bounded interval $\left(0, t_{\text {obs }}\right]$. To see how good this approximation is, we consider the two-norm of the vector $R_{m}(t)$ as a function of $t$ for $t>0$.

First of all, it is observed that since $0<\tau<t$ in (30), we have

$$
\|\exp [-A(t-\tau)]\| \leq \exp [\|A\|(t-\tau)] \leq \exp (\|A\| t)
$$

and the same inequality is true if we replace matrix $A$ by matrix $T_{m}$. Taking the two-norm of the vector $R_{m}(t)$ and using the result of (33) leads to

$$
\begin{aligned}
\left\|R_{m}(t)\right\| & \leq\|Q\| \frac{(\|A\| t)^{m}}{m !} \exp (\|A\| t) \\
& +\left|\beta_{1}\right|\left\|V_{m}\right\| \frac{\left(\left\|T_{m}\right\| t\right)^{m}}{m !} \exp \left(\left\|T_{m}\right\| t\right)
\end{aligned}
$$

and for $m \rightarrow \infty$ this can be written as

$$
\begin{aligned}
\left\|R_{m}(t)\right\| & \leq\|Q\|\left(\frac{\|A\| t e}{m}\right)^{m} \exp (\|A\| t) \\
& +\left|\beta_{1}\right|\left\|V_{m}\right\|\left(\frac{\left\|T_{m}\right\| t e}{m}\right)^{m} \exp \left(\left\|T_{m}\right\| t\right)
\end{aligned}
$$

where we have used Stirling's formula for $m$ !. We observe that $\left\|R_{m}(t)\right\|$ becomes negligible as soon as $m>t e s_{0}$, where $s_{0}$ is given by (25), under the assumption that $\left\|V_{m}\right\|$ does not become too large. In order to have reliable results on the interval of observation $\left(0, t_{\mathrm{obs}}\right]$, we must have $t_{\mathrm{obs}}<m /\left(e s_{0}\right)$ showing that the length of this interval is linearly related to the number of Lanczos steps.

\section{A. Computational Considerations}

The reduced-model approximation is computed as follows. From Appendix A, we know that in the lossless case, matrix $T_{m}$ is diagonalizable. Now let $T_{m} S_{m}=S_{m} \Theta_{m}$ denote the eigenvalue problem for matrix $T_{m}$, then the expression for $F_{m}(t)$ can be written as

$$
F_{m}(t)=w(t) * \beta_{1} M_{2}^{-1} V_{m} S_{m} \exp \left(-\Theta_{m} t\right) S_{m}^{T} e_{1} .
$$

This expression is computed for field quantities in the observation points of interest. This means that only rows of matrix $V_{m}$ which correspond to these field quantities are kept in memory. Note that solving the eigenvalue problem for matrix $T_{m}$ can be done by standard eigenvalue problem routines, since $m$ is very small, especially compared to $N$ and, moreover, matrix $T_{m}$ is tridiagonal and symmetric. Also note the important role that the first components of the eigenvectors of matrix $T_{m}$ play in forming the reduced-model approximation.

When losses are involved, we no longer a priori know if matrix $T_{m}$ is diagonalizable. Instead, we then assume that it is diagonalizable. We do mention that in all of our present numerical work, we have never detected a case where matrix $T_{m}$ was not diagonalizable. Also, from the characteristic polynomial of matrix $T_{m}$, it is observed that all eigenvalues of matrix $T_{m}$ are either real or occur in complex conjugate pairs. The same is true for the eigenvalues of matrix $A$. Moreover, it is easily seen that all eigenvalues of matrix $A$ have a nonnegative real part. However, this can not be said of the eigenvalues of matrix $T_{m}$, i.e., it may happen that some eigenvalues of matrix $T_{m}$ have a negative real part. These eigenvalues are obviously located at the wrong half of the complex plane. This phenomenon occurs only when losses are involved. In the lossless case, we have a standard Lanczos algorithm which is known to produce stable results [12]. In our numerical tests, we further have observed the fact that for eigenvalues of matrix $T_{m}$ with a substantial negative real part, the absolute value of the first component of its corresponding eigenvector becomes negligible. The larger the absolute value of the negative real part of an eigenvalue is, the smaller the absolute value of the first component of its corresponding eigenvector. Our approach is, therefore, to shift all eigenvalues with a negative real part onto the imaginary axis. This has the effect that eigenvalues with a substantial negative real part do not contribute to the reduced-model approximation as is indicated by the algorithm by means of a vanishing first component of its corresponding eigenvector. From the numerical results we have observed that the error of this approach, if any, falls below the discretization error.

Since our Lanczos algorithm employs a bilinear form and not an inner product, it may happen that $\beta_{i+1}=0$ even when $w_{i}-\alpha_{i} v_{i} \neq 0$. The algorithm cannot continue in such a case. This is called breakdown of the algorithm. In particular, there exist source vectors $Q$ for which $\beta_{1}=0$ and so it is not even possible to start the iteration process for such source vectors. It must be said, however, that these source vectors always consist of a part due to external electric-current sources and a part due to external magnetic-current sources. Carrying out the modified Lanczos algorithm with such a source vector would 
imply that we are interested in the situation where we have two different kinds of external sources, one electric the other magnetic, but with the same source wavelet $w(t)$. Obviously, this is a rather exceptional situation. Nevertheless, if one is interested in the response due to such a source setup, one first computes the response due to the external electric-current sources, then one computes the response due to the external magnetic-current sources and finally one uses the superposition principle. Such an approach is possible due to the linearity of the problem and because of the fact that a source vector with an external electric-current part only, or with an external magnetic-current part only, can not result in a $\beta_{1}=0$; i.e., we can always start. Furthermore, in Appendix A, it is shown that in case of a lossless medium and a source vector with either an electric-current part or a magnetic-current part, breakdown of the algorithm does not occur. Although in our present numerical work we have never detected a breakdown of the algorithm in case of lossy media, presently we are not able to prove that in the lossy case, theoretically no breakdown occurs.

\section{Numerical Results}

We have implemented the reduced-model technique for 2-D $E$ - or $H$-polarized waves in inhomogeneous, isotropic, and lossy media. The configuration is invariant in the $x_{2}$-direction and the $x_{3}$-direction is chosen downwardly. The presented results are all for the case of $E$-polarization (electric field strength $E_{2}$ parallel to the invariance direction), except for the last example where, for completeness, the case of $\mathrm{H}$ polarization is given as well. In all examples the source vector is of the electric-current type, i.e., the source vector $Q$ satisfies $Q=\delta^{\mathrm{E}} Q$. The source wavelet is taken to be a Ricker wavelet and is given by

$$
w(t)=\chi(t) \sqrt{\frac{e}{2 \theta}} \frac{d}{d t} \exp \left[-\theta\left(t-t_{0}\right)^{2}\right] .
$$

With the parameter $t_{0}$, we can shift the nonzero part of the wavelet in time and by varying the parameter $\theta$, we can vary the peak frequency of this wavelet. In all examples, $\theta$ is chosen such that this frequency is $40 \mathrm{MHz}$. We employ a uniform mesh with a meshwidth such that we have about 34 points $/ \lambda$, where $\lambda$ is the free-space wavelength corresponding to the peak frequency of $40 \mathrm{MHz}$. For this mesh, the order $N$ of matrix $A$ amounts to 189000 .

As a first example, we consider the electric-field strength $E_{2}=E_{2}\left(x_{1}, x_{3}, t\right)$ in a vacuum domain generated by an external electric-current source of the type $J_{1}^{\mathrm{e}}\left(x_{1}, x_{3}, t\right)=0$, $J_{2}^{\mathrm{e}}\left(x_{1}, x_{3}, t\right)=w(t) \delta\left(x_{1}, x_{3}\right), J_{3}^{\mathrm{e}}\left(x_{1}, x_{3}, t\right)=0$. For this particular example, the electric-field strength is known in closed form as

$$
E_{2}\left(x_{1}, x_{3}, t\right)= \begin{cases}0, & t<T \\ -\frac{\mu_{0}}{2 \pi} \int_{\tau=T}^{t} \frac{\partial_{t} w(t-\tau)}{\sqrt{\tau^{2}-T^{2}}} d \tau, & t>T\end{cases}
$$

where $T=\left(x_{1}^{2}+x_{3}^{2}\right)^{1 / 2} / c_{0}$ is the arrival time for the wave to travel from the source location to the observation point, $c_{0}$ is the electromagnetic-wave speed in vacuum and $\mu_{0}$ is the permeability of vacuum. In our finite-difference

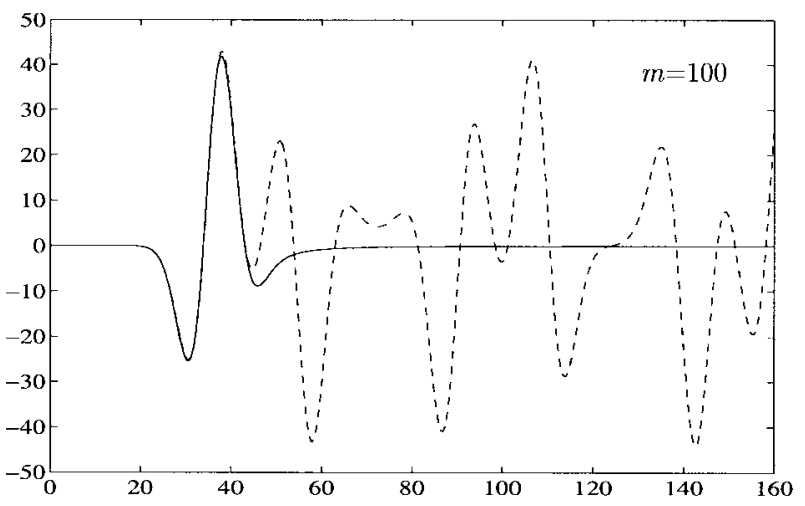

(a)

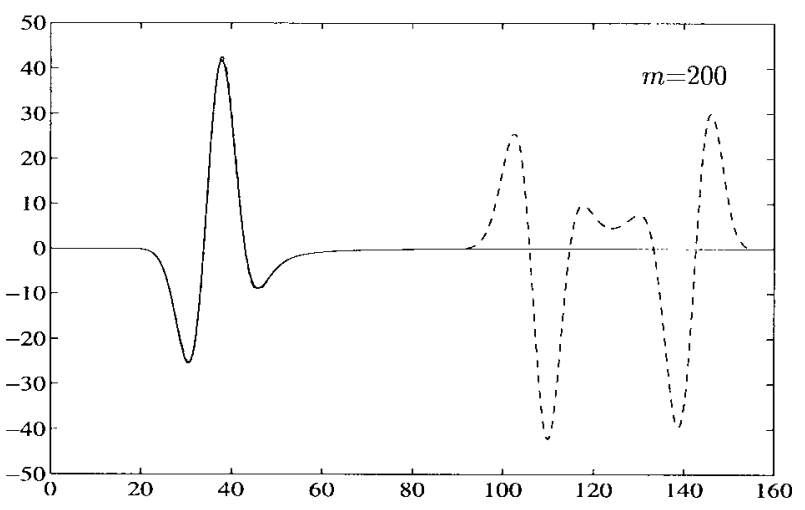

(b)

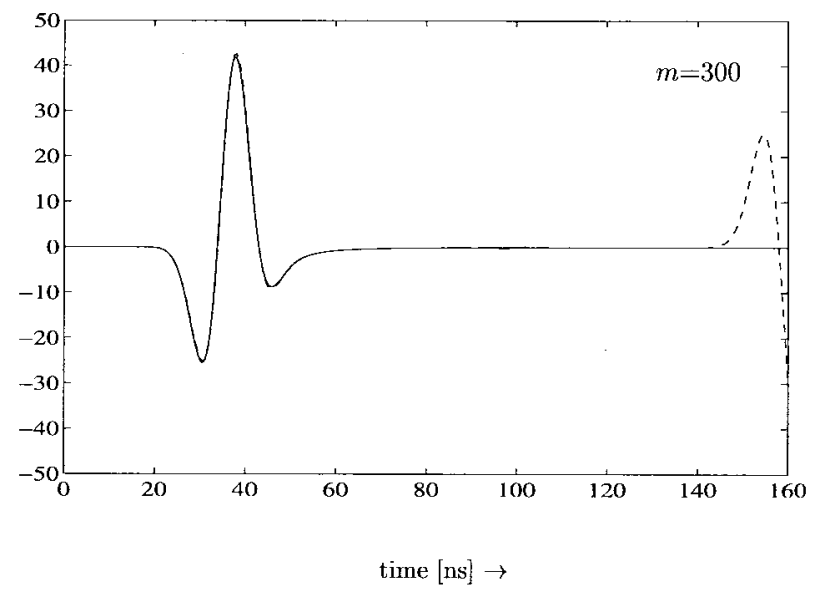

(c)

Fig. 2. Electric field strength $E_{2}$ as a function of time as measured by the receiver in a vacuum domain. The solid line signifies the exact result. The dashed line is the reduced-model approximation (a) after 100 Lanczos iterations, (b) after 200 iterations, and (c) after 300 iterations.

approximation, the delta function $\delta\left(x_{1}, x_{3}\right)$ is approximated by a 2-D triangular distribution. The expression for $E_{2}$ [as given by (38)] is, therefore, weighted over this distribution. For an observation point located $4.84 \mathrm{~m}$ from the source, we then obtain the result as given by the solid line in Fig. 2. The dashed line in Fig. 2(a) is the reduced-model approximation after 100 Lanczos steps, in Fig. 2(b) after 200 Lanczos steps, and in Fig. 2(c) after 300 Lanczos steps. We observe that the length of the interval in which the reduced-model approximation and 


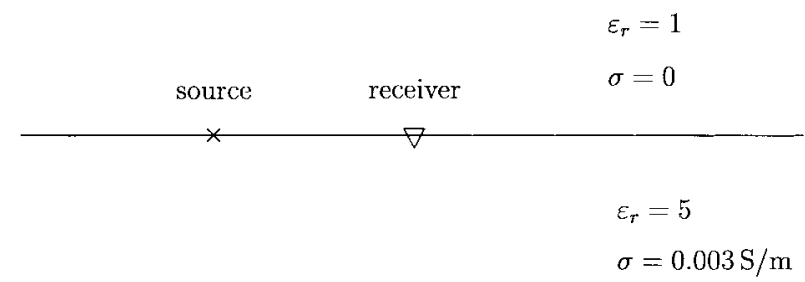

Fig. 3. Source and receiver located at the interface of a lossy half-space. The distance between the source and the receiver is $4.84 \mathrm{~m}$.

the exact result overlap, increases with the number of Lanczos steps.

As a second example, we consider the same source-receiver setup, but now located at the interface of a lossy half-space (see Fig. 3). The solid line in Fig. 4 is the result obtained after 650 Lanczos steps. The dashed line in Fig. 4(a) is the result after 200 Lanczos steps, in Fig. 4(b) after 400 Lanczos steps, and in Fig. 4(c) after 600 Lanczos steps. Note the different scale in Fig. 4(a). Again, the same behavior as in the previous example is observed. However, it takes more Lanczos steps to get an accurate approximation on the same time interval when compared to the case of a vacuum domain.

As a third example, we show the results obtained when an object is present in the lossy half-space (see Fig. 5). The source and receiver are symmetrically located above the object. The configuration shown in Fig. 5 is similar to the 3-D configuration as taken by Wang and Tripp [13]. The solid line in Fig. 6 is the result obtained after 650 Lanczos steps and the dashed lines show the reduced-model approximations after 200 Lanczos steps [Fig. 6(a)], 400 Lanczos steps [Fig. 6(b)], and 600 Lanczos steps [Fig. 6(c)]. Note the different scale in Fig. 6(a). The presence of the buried object is clearly seen in the time interval 70-160 ns. In Fig. 7, we show the results for the configuration of Fig. 5 in case of $H$ polarized waves excited by an external electric-current source of the type $J_{1}^{\mathrm{e}}\left(x_{1}, x_{3}, t\right)=w(t) \delta\left(x_{1}, x_{3}\right), J_{2}^{\mathrm{e}}\left(x_{1}, x_{3}, t\right)=0$, $J_{3}^{\mathrm{e}}\left(x_{1}, x_{3}, t\right)=0$.

\section{B. Comparison with the FDTD Method}

Consider again, the configuration of Fig. 5. In Fig. 8, we repeat (for the case of $E$-polarization), the result obtained by the modified Lanczos algorithm after 650 Lanczos steps (solid line) and we show the result obtained via the FDTD method using the same uniform mesh in space as in the reducedmodel technique and using a time step of $\Delta t=\Delta x /\left(2 c_{0}\right)$ (dashed line). The results show a good overall agreement, but the FDTD result deviates slightly from the reduced-model approximation. To study this deviation a bit further, we have plotted the reduced-model approximation and the FDTD result in more detail for time steps of $\Delta t=\Delta x /\left(\sqrt{2} c_{0}\right), \Delta t=$ $\Delta x /\left(2 c_{0}\right)$, and $\Delta t=\Delta x /\left(4 c_{0}\right)$ from about $t=70 \mathrm{~ns}$ up to $t=80 \mathrm{~ns}$ in Fig. 9. We see that by decreasing the time step, the FDTD result converges to the reduced-model approximation. For a time step of $\Delta t=\Delta x /\left(8 c_{0}\right)$, the FDTD result and the reduced-model approximation completely coincide.

As a second comparison, we consider the same source as in the previous examples and a receiver located $0.44 \mathrm{~m}$ from

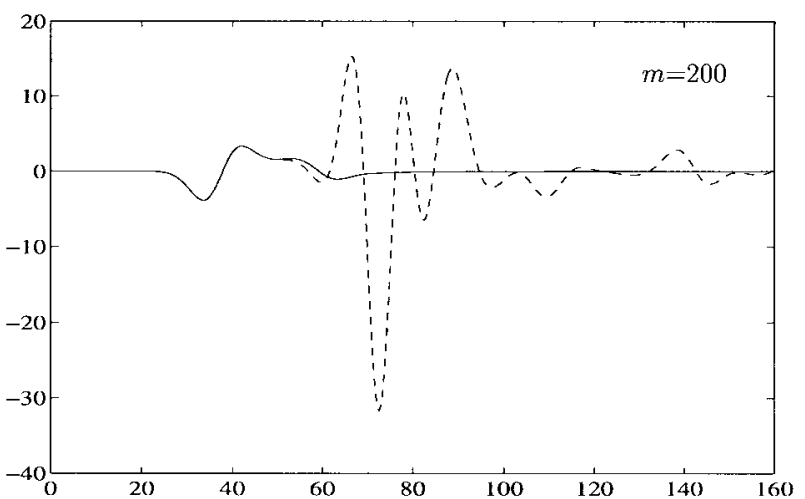

(a)

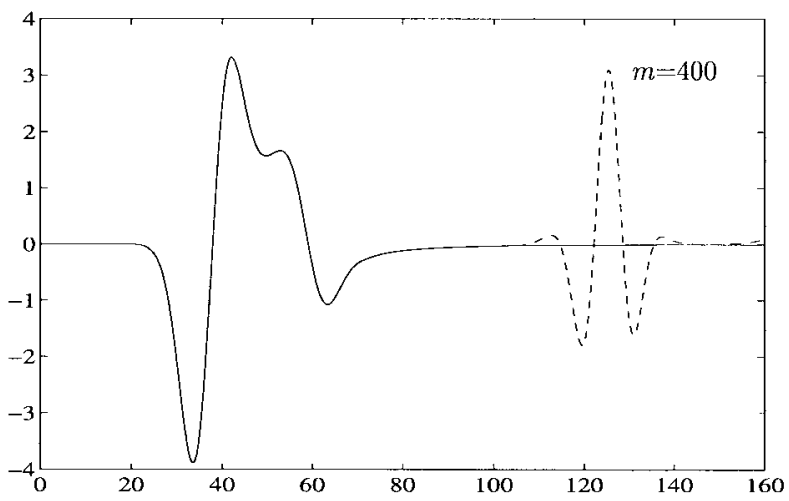

(b)

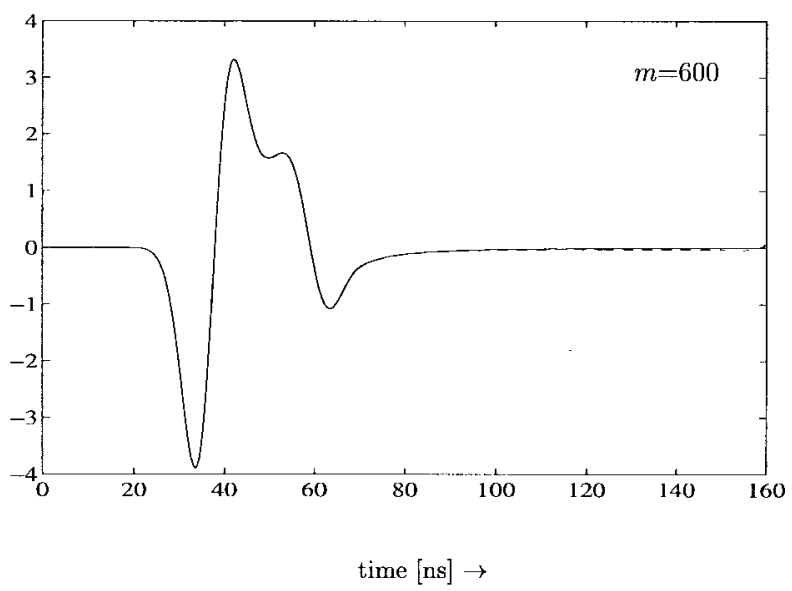

(c)

Fig. 4. Electric-field strength $E_{2}$ as a function of time as measured by the receiver in the configuration of Fig. 3. The solid line is the reduced-model approximation after 650 Lanczos steps. The dashed line is the reduced-model approximation (a) after 200 Lanczos steps, (b) after 400 Lanczos steps, and (c) after 600 Lanczos steps.

the source. Fig. 10(a) shows the FDTD result with a time step of $\Delta t=\Delta x /\left(2 c_{0}\right)$ in case of a vacuum domain. If we now take the same source-receiver setup and the same spatial and temporal discretization in a homogeneous and lossy medium of $\sigma=4 \mathrm{~S} / \mathrm{m}$, we get the FDTD result, as shown in Fig. 10(b). The numerical dispersion due to the time discretization is clearly observed. Using a time step of 


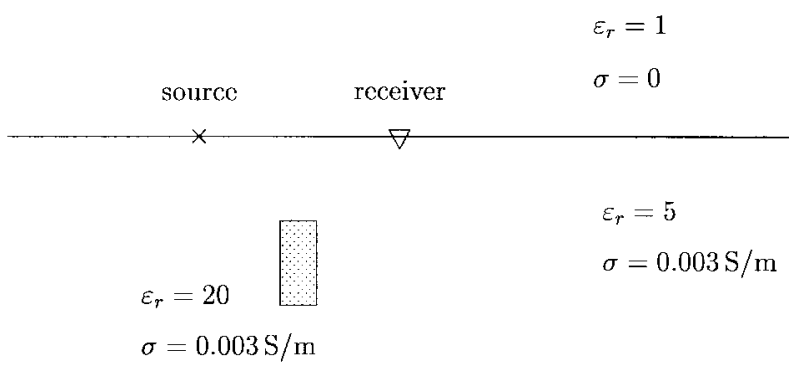

Fig. 5. Source and receiver located at the interface of a lossy half-space with a buried object of $0.88 \mathrm{~m} \times 1.98 \mathrm{~m}$. The distance between the source and the receiver is $4.84 \mathrm{~m}$. The top of the object is located $1.98 \mathrm{~m}$ below the interface.

$\Delta t=\Delta x /\left(4 c_{0}\right)$ gives the result as shown by the solid line in Fig. 10(c). From these results, we observe that the dispersion effect disappears if the time step is chosen sufficiently small. Also shown in Fig. 10(c) is the reduced model obtained after six Lanczos steps (dashed line) and after 12 Lanczos steps (solid line). The reduced model obtained after 12 Lanczos steps completely coincides with the FDTD result using a time step of $\Delta t=\Delta x /\left(4 c_{0}\right)$. From this result, we conclude that after only 12 steps, the reduced model is already accurate for this configuration. From a computational point of view, one step of the FDTD method is approximately equal to one step of the modified Lanczos algorithm and so, especially in this last example, the computational savings can be substantial when the reduced-model technique is being used.

\section{CONCLUSIONS}

In this paper, we have presented a new method for computing transient electromagnetic wavefields in inhomogeneous media. The cornerstone of the method is a so-called reduced model that gives an accurate representation of the transient electromagnetic-wave field on a certain bounded interval in time. The length of this interval can be extended by performing more steps of the underlying Lanczos algorithm. One of the characteristics of the method is that it is not an explicit timestepping method and, therefore, errors introduced due to the discretization of the time variable are not observed.

Although we have presented the numerical results for 2-D configurations, the theory applies to the 3-D case as well. Future work will concentrate on this 3-D case as well as on the implementation of absorbing-boundary conditions. The only candidates that can be used in this method are the absorbingboundary conditions based on the physics of the problem; e.g., the anisotropic absorbers introduced by Sacks et al. [14] and the absorbers as proposed by Ziolkowski [15].

The reduced model is constructed by employing the method of Lanczos. This is a Krylov subspace method and the convergence rate of such methods can be accelerated by using suitable preconditioning techniques. In our case of computing transient electromagnetic-wave fields, we can use techniques similar to the ones presented in [16]. Although the reducedmodel technique is a reasonable efficient method in itself, it is to be expected that these preconditioning techniques will dramatically improve the convergence rate of the modified Lanczos algorithm.

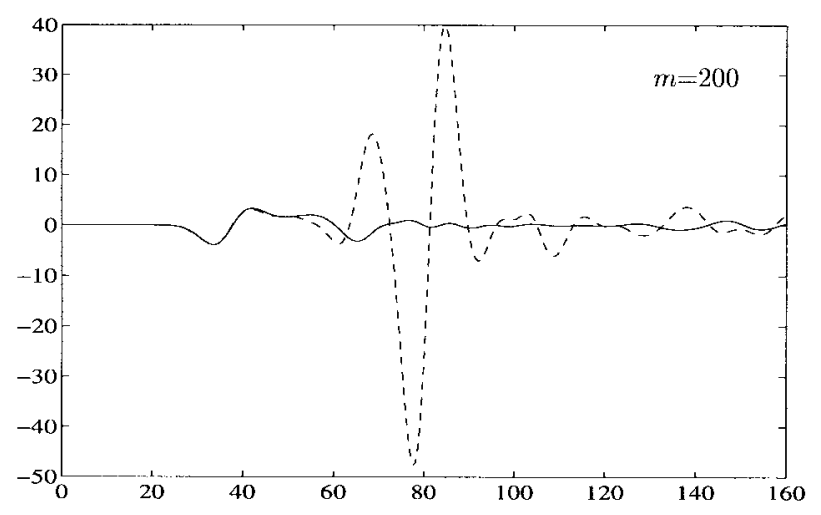

(a)

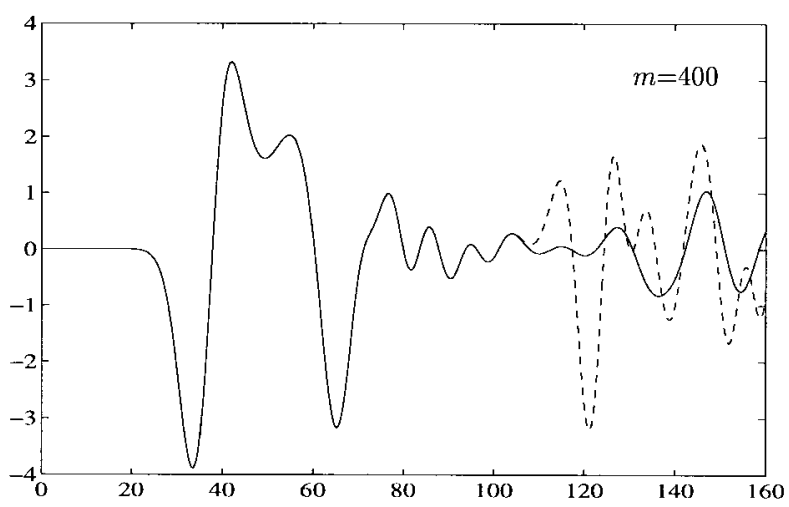

(b)

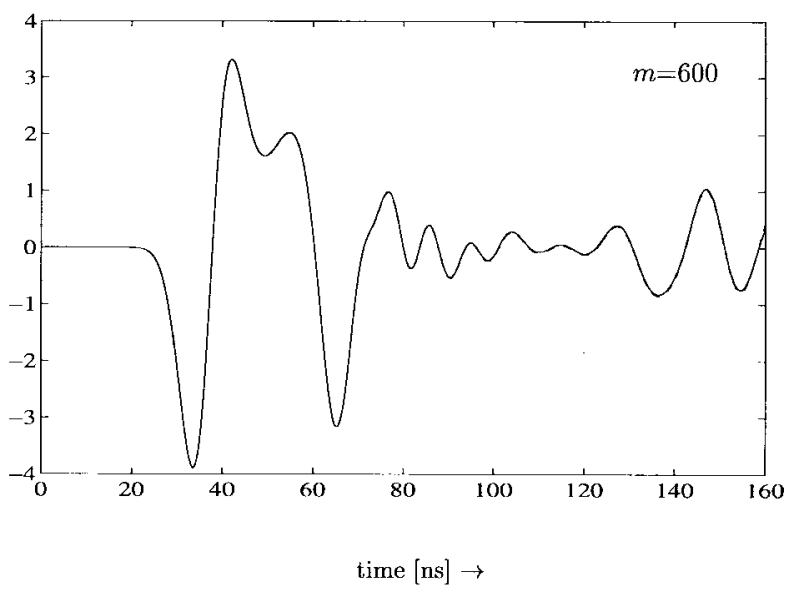

(c)

Fig. 6. Electric-field strength $E_{2}$ as a function of time as measured by the receiver in the configuration of Fig. 5. Solid line is the reduced-model approximation after 650 Lanczos steps. Dashed line is the reduced-model approximation (a) after 200 Lanczos steps, (b) after 400 Lanczos steps, and (c) after 600 Lanczos steps (c).

\section{APPENDIX A}

Here we study the behavior of the modified Lanczos algorithm in case of a lossless medium.

Let us define, in addition to the signature matrix $\delta^{-}$, the identity matrix $\delta^{+}$and the matrices $\delta^{E}$ and $\delta^{H}$ as

$$
\begin{aligned}
& \delta^{+}=\operatorname{diag}(1,1,1,1,1,1), \\
& \delta^{E}=\frac{1}{2}\left(\delta^{+}+\delta^{-}\right)=\operatorname{diag}(1,1,1,0,0,0)
\end{aligned}
$$




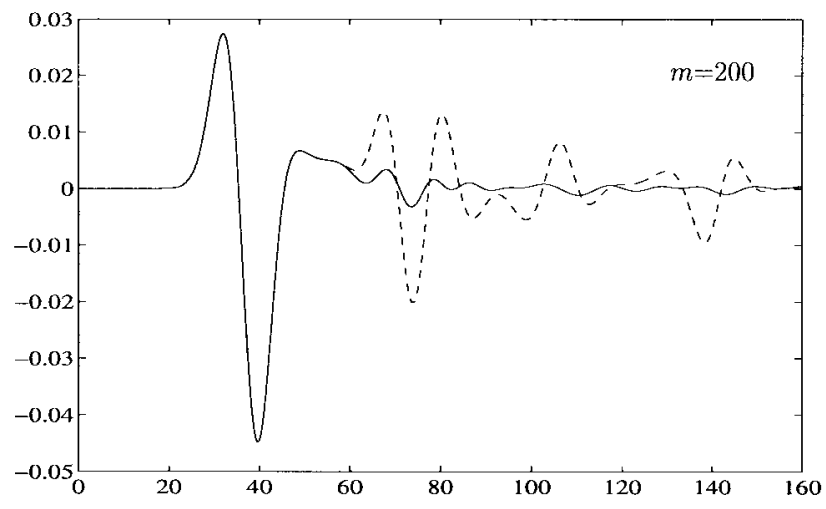

(a)
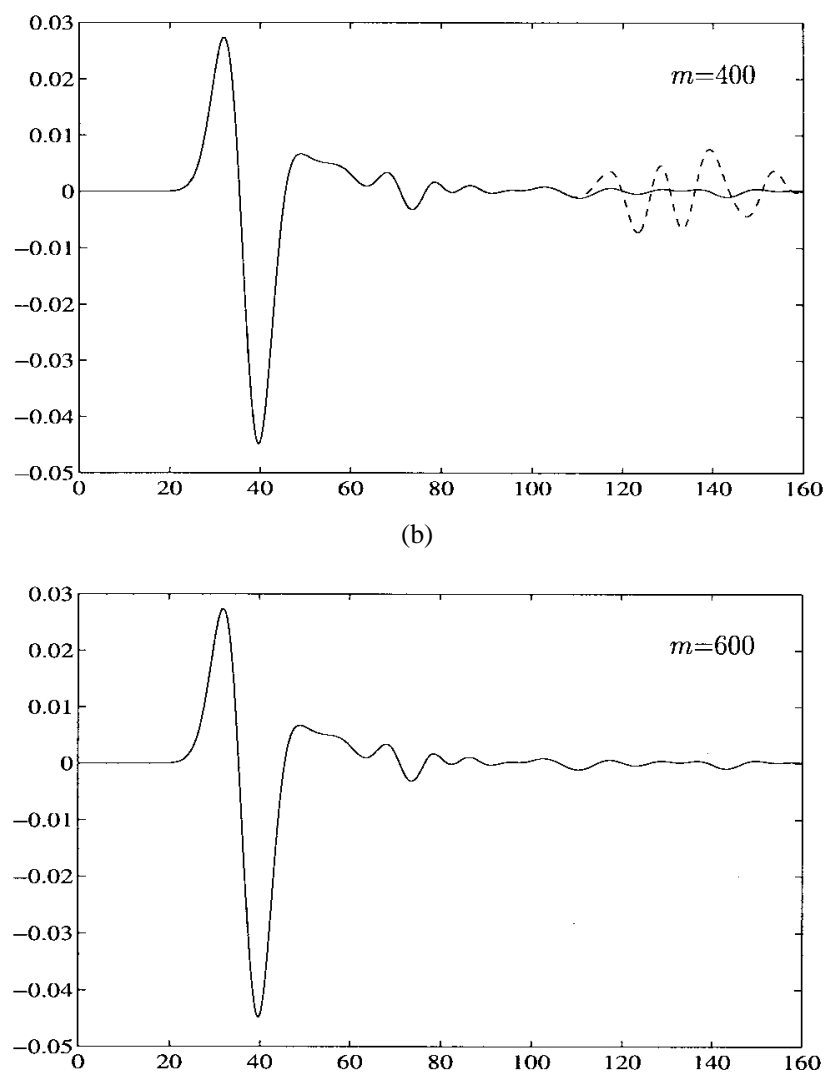

time $[\mathrm{ns}] \rightarrow$

(c)

Fig. 7. Magnetic-field strength $\mathrm{H}_{2}$ as a function of time as measured by the receiver in the configuration of Fig. 5. The solid line is the reduced-model approximation after 650 Lanczos steps. The dashed line is the reduced-model approximation (a) after 200 Lanczos steps, (b) after 400 Lanczos steps, and (c) after 600 Lanczos steps.

and

$$
\delta^{H}=\frac{1}{2}\left(\delta^{+}-\delta^{-}\right)=\operatorname{diag}(0,0,0,1,1,1) .
$$

From (8) and the fact that the identity matrix $\delta^{+}$commutes with matrix $\mathcal{D}$, it follows that

$$
\mathcal{D} \delta^{E}=\delta^{H} \mathcal{D}
$$

and

$$
\mathcal{D} \delta^{H}=\delta^{E} \mathcal{D}
$$

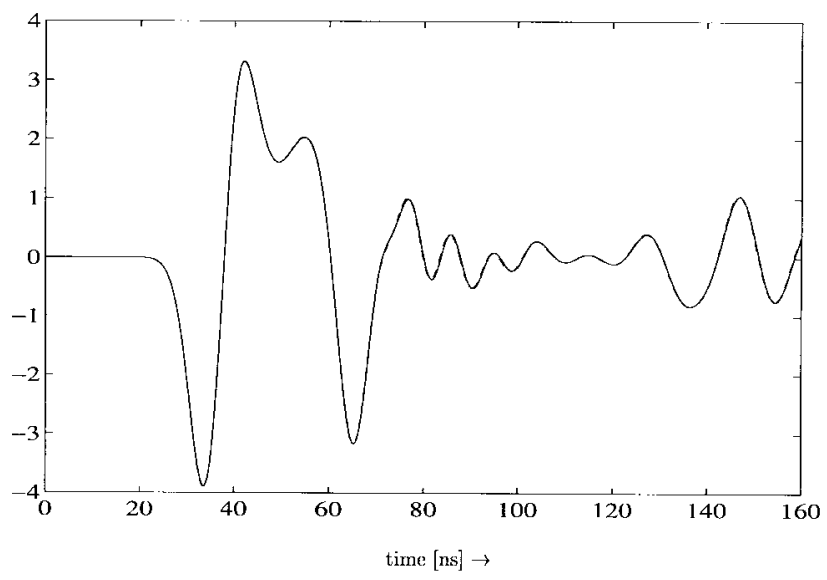

Fig. 8. Electric-field strength $E_{2}$ as a function of time as measured by the receiver in the configuration of Fig. 5. The solid line is the reduced-model approximation after 650 Lanczos steps. The dashed line is the FDTD result with a time step of $\Delta t=\Delta x /\left(2 c_{0}\right)$.

Equations (42) and (43) show that when $\mathcal{D}$ operates on a vector proportional to the electric-field strength, a vector proportional to the magnetic-field strength results, and vice versa. This can also be seen from the block off-diagonal structure of matrix $\mathcal{D}$.

Other useful relations are

$$
\begin{aligned}
& \delta^{-} \delta^{E}=\delta^{E} \\
& \delta^{-} \delta^{H}=-\delta^{H} .
\end{aligned}
$$

The discrete versions of $\delta^{E}$ and $\delta^{H}$ are also denoted by $\delta^{E}$ and $\delta^{H}$. Equations (42)-(45) each have their discrete counterpart. Apart from the relations already mentioned, we also use the relations

$$
M_{2}^{-1} \delta^{E}=\delta^{E} M_{2}^{-1}
$$

and

$$
M_{2}^{-1} \delta^{H}=\delta^{H} M_{2}^{-1}
$$

These equations follow from the fact that matrix $M_{2}$ commutes with the matrices $\delta^{E}$ and $\delta^{H}$. We have the following theorem, in which $\mathcal{I}$ denotes the imaginary axis, characterizing the modified Lanczos algorithm in case of a lossless medium.

Theorem 2: If the real-valued source vector $Q$ satisfies $Q=$ $\delta^{E} Q$, i.e., no external magnetic-current sources are present, then the coefficients of the modified Lanczos algorithm satisfy

$$
\begin{aligned}
& \alpha_{i}=0, \quad i=1,2, \cdots \\
& \beta_{i} \in \mathcal{I} \backslash\{0\}, \quad i=2,3, \cdots
\end{aligned}
$$

and the Lanczos vectors generated by this algorithm satisfy

$$
v_{i}= \begin{cases}\delta^{E} v_{i}, & \text { a real-valued vector when } i \text { is odd } \\ \delta^{H} v_{i}, & \text { an imaginary-valued vector when } i \text { is even. }\end{cases}
$$

Furthermore, breakdown of the algorithm cannot occur.

Proof: From the condition $\left\langle v_{1}, v_{1}\right\rangle_{\mathrm{b}}=1$, we have

$$
\begin{aligned}
\left\langle v_{1}, v_{1}\right\rangle_{\mathrm{b}} & =\beta_{1}^{-2}\langle Q, Q\rangle_{\mathrm{b}}=\beta_{1}^{-2}\left\langle M_{2}^{-1} \delta^{-} Q, Q\right\rangle \\
& =\beta_{1}^{-2}\left\langle M_{2}^{-1} \delta^{-} \delta^{E} Q, Q\right\rangle=\beta_{1}^{-2}\left\langle M_{2}^{-1} Q, Q\right\rangle=1
\end{aligned}
$$




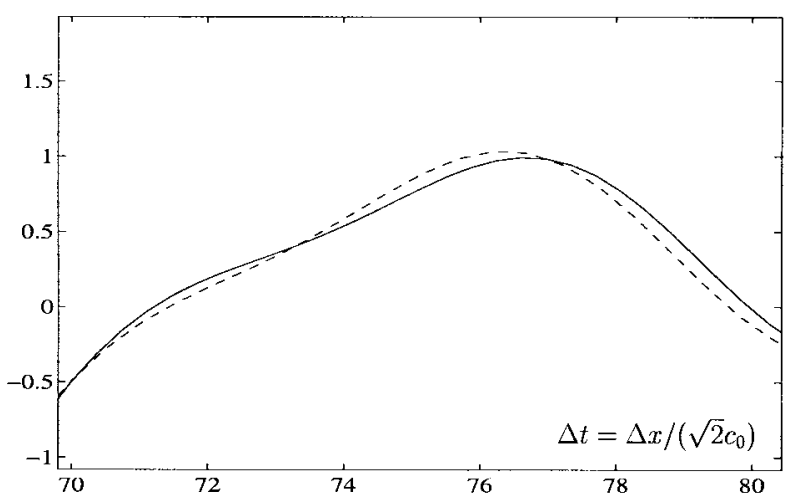

(a)

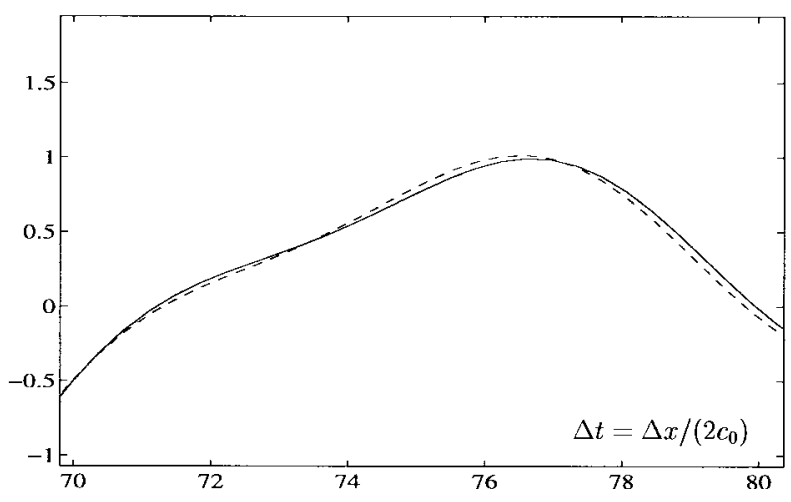

(b)

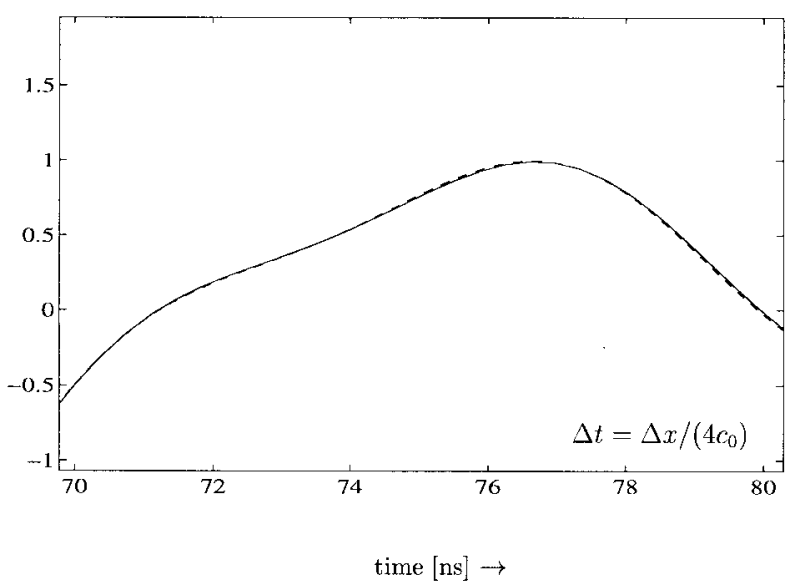

(c)

Fig. 9. Detail of the electric-field strength $E_{2}$ of Fig. 8. The solid line is the reduced-model approximation after 650 Lanczos steps. The dashed line is the FDTD result with a time step of (a) $\Delta t=\Delta x /\left(\sqrt{2} c_{0}\right)$, (b) $\Delta t=\Delta x /\left(2 c_{0}\right)$, and (c) $\Delta t=\Delta x /\left(4 c_{0}\right)$.

and so

$$
\beta_{1}^{2}=\left\langle M_{2}^{-1} Q, Q\right\rangle>0
$$

showing that $\beta_{1}$ is real and not equal to zero. From the relation $\beta_{1} v_{1}=Q$ it follows that the vector $v_{1}$ is real-valued and satisfies $v_{1}=\delta^{E} v_{1}$. The rest of the proof follows by induction over $i$.

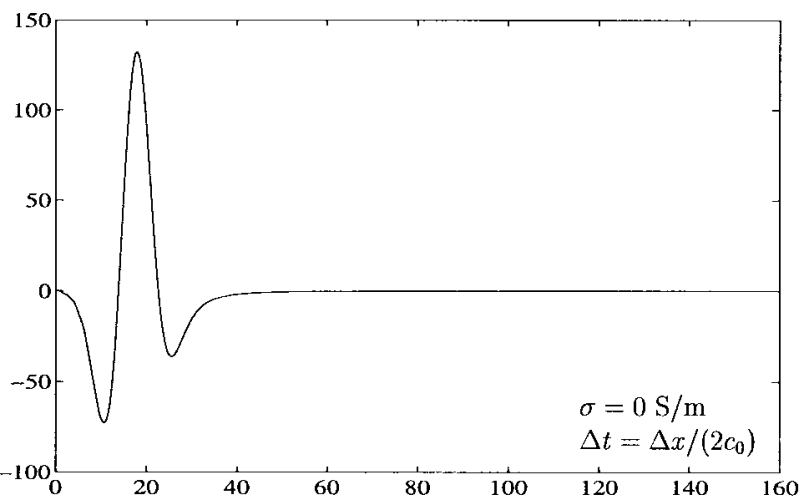

(a)

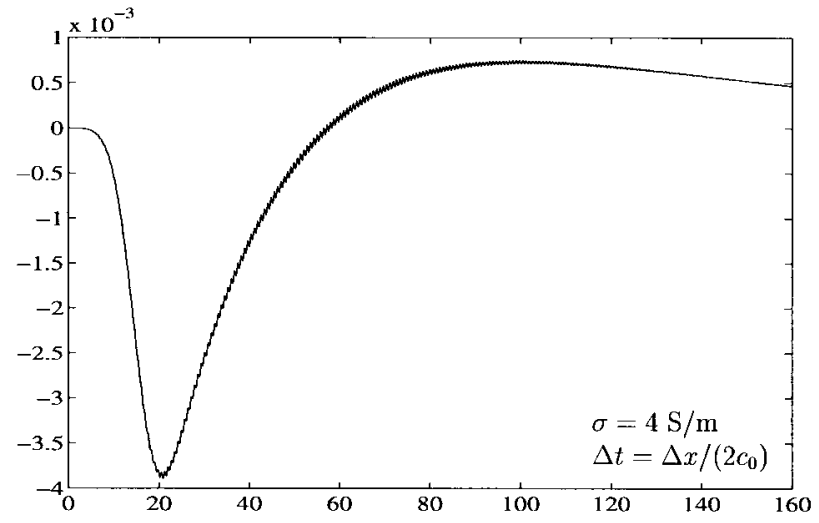

(b)

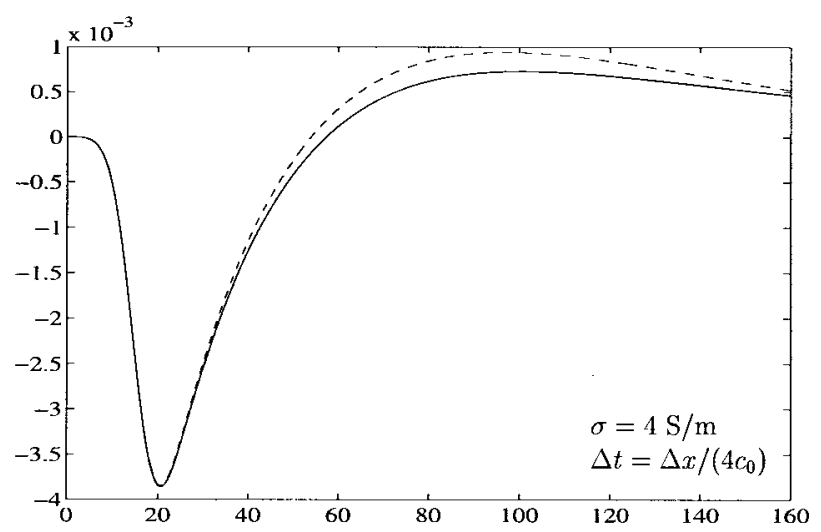

time $[\mathrm{ns}] \rightarrow$

(c)

Fig. 10. Electric-field strength $E_{2}$ as a function of time as measured by the receiver in a homogeneous space. (a) FDTD result with a time step of $\Delta t=\Delta x /\left(2 c_{0}\right)$ in case of a vacuum domain. (b) FDTD result in case $\sigma=4 \mathrm{~S} / \mathrm{m}$ using the same time step. (c) FDTD result (solid line) with a time step of $\Delta t=\Delta x /\left(4 c_{0}\right)$ and the reduced-model approximation after six Lanczos steps (dashed line). The reduced-model approximation after 12 Lanczos steps coincides with the FDTD result.

For $i=1$ we have

$$
\begin{aligned}
\alpha_{1} & =\left\langle v_{1}, w_{1}\right\rangle_{\mathrm{b}}=\left\langle v_{1}, D M_{2}^{-1} v_{1}\right\rangle_{\mathrm{b}} \\
& =\left\langle M_{2}^{-1} \delta^{-} v_{1}, D M_{2}^{-1} v_{1}\right\rangle=\left\langle M_{2}^{-1} \delta^{-} \delta^{E} v_{1}, D M_{2}^{-1} v_{1}\right\rangle \\
& =\left\langle M_{2}^{-1} v_{1}, D M_{2}^{-1} v_{1}\right\rangle=0
\end{aligned}
$$


since $D$ is skew-symmetric. This leaves us with

$$
\begin{aligned}
\beta_{2} v_{2} & =w_{1}=D M_{2}^{-1} v_{1}=D M_{2}^{-1} \delta^{E} v_{1}=D \delta^{E} M_{2}^{-1} v_{1} \\
& =\delta^{H} D M_{2}^{-1} v_{1}=\delta^{H} w_{1} .
\end{aligned}
$$

The vector $w_{1}$ is a real-valued vector satisfying $w_{1}=\delta^{H} w_{1}$. From the condition $\left\langle v_{2}, v_{2}\right\rangle_{\mathrm{b}}=1$, we have

$$
\begin{aligned}
\left\langle v_{2}, v_{2}\right\rangle_{\mathrm{b}} & =\beta_{2}^{-2}\left\langle w_{1}, w_{1}\right\rangle_{\mathrm{b}}=\beta_{2}^{-2}\left\langle M_{2}^{-1} \delta^{-} w_{1}, w_{1}\right\rangle \\
& =-\beta_{2}^{-2}\left\langle M_{2}^{-1} w_{1}, w_{1}\right\rangle=1
\end{aligned}
$$

and so

$$
\beta_{2}^{2}=-\left\langle M_{2}^{-1} w_{1}, w_{1}\right\rangle<0 \text { for a nonzero } w_{1} .
$$

This shows that $\beta_{2} \in \mathcal{I} \backslash\{0\}$. Since $v_{2}=\beta_{2}^{-1} w_{1}, v_{2}$ is an imaginary-valued vector satisfying $v_{2}=\delta^{H} v_{2}$.

Assume that $i$ is odd. Then, by the induction hypothesis, $v_{i}$ is a real-valued vector satisfying $v_{i}=\delta^{E} v_{i}, v_{i-1}$ is an imaginary-valued vector satisfying $v_{i-1}=\delta^{H} v_{i-1}, \beta_{i}$ is imaginary and not equal to zero and $\alpha_{i}=0$. We now have

$$
\begin{aligned}
\beta_{i+1} v_{i+1} & =w_{i}=D M_{2}^{-1} v_{i}-\beta_{i} v_{i-1} \\
& =D M_{2}^{-1} \delta^{E} v_{i}-\beta_{i} \delta^{H} v_{i-1} \\
& =D \delta^{E} M_{2}^{-1} v_{i}-\beta_{i} \delta^{H} v_{i-1} \\
& =\delta^{H}\left(D M_{2}^{-1} v_{i}-\beta_{i} v_{i-1}\right)=\delta^{H} w_{i} .
\end{aligned}
$$

The vector $w_{i}$ is a real-valued vector satisfying $w_{i}=\delta^{H} w_{i}$. From the condition that $\left\langle v_{i+1}, v_{i+1}\right\rangle_{\mathrm{b}}=1$, we have

$$
\begin{aligned}
\left\langle v_{i+1}, v_{i+1}\right\rangle_{\mathrm{b}} & =\beta_{i+1}^{-2}\left\langle w_{i}, w_{i}\right\rangle_{\mathrm{b}}=\beta_{i+1}^{-2}\left\langle M_{2}^{-1} \delta^{-} w_{i}, w_{i}\right\rangle \\
& =\beta_{i+1}^{-2}\left\langle M_{2}^{-1} \delta^{-} \delta^{H} w_{i}, w_{i}\right\rangle \\
& =-\beta_{i+1}^{-2}\left\langle M_{2}^{-1} w_{i}, w_{i}\right\rangle=1
\end{aligned}
$$

and so

$$
\beta_{i+1}^{2}=-\left\langle M_{2}^{-1} w_{i}, w_{i}\right\rangle<0 \text { for a nonzero } w_{i} .
$$

This shows that $\beta_{i+1} \in \mathcal{I} \backslash\{0\}$. Since $v_{i+1}=\beta_{i+1}^{-1} w_{i}$, $v_{i+1}$ is an imaginary-valued vector satisfying $v_{i+1}=\delta^{H} v_{i+1}$. Further,

$$
\begin{aligned}
\alpha_{i+1} & =\left\langle v_{i+1}, w_{i+1}\right\rangle_{\mathrm{b}}=\left\langle v_{i+1}, D M_{2}^{-1} v_{i+1}\right\rangle_{\mathrm{b}} \\
& =\left\langle M_{2}^{-1} \delta^{-} v_{i+1}, D M_{2}^{-1} v_{i+1}\right\rangle \\
& =-\left\langle M_{2}^{-1} v_{i+1}, D M_{2}^{-1} v_{i+1}\right\rangle=0 .
\end{aligned}
$$

As a next step, we assume that $i$ is even. Then, by the induction hypothesis, $v_{i}$ is an imaginary-valued vector satisfying $v_{i}=\delta^{H} v_{i}, v_{i-1}$ is a real-valued vector satisfying $v_{i-1}=\delta^{E} v_{i-1}, \beta_{i}$ is imaginary and not equal to zero and $\alpha_{i}=0$. We now have

$$
\begin{aligned}
\beta_{i+1} v_{i+1} & =w_{i}=D M_{2}^{-1} v_{i}-\beta_{i} v_{i-1} \\
& =D M_{2}^{-1} \delta^{H} v_{i}-\beta_{i} \delta^{E} v_{i-1} \\
& =\delta^{E}\left(D M_{2}^{-1} v_{i}-\beta_{i} v_{i-1}\right)=\delta^{E} w_{i} .
\end{aligned}
$$

The vector $w_{i}$ is an imaginary-valued vector satisfying $w_{i}=$ $\delta^{E} w_{i}$. From the condition that $\left\langle v_{i+1}, v_{i+1}\right\rangle_{\mathrm{b}}=1$ it follows that

$$
\beta_{i+1}^{2}=\left\langle M_{2}^{-1} w_{i}, w_{i}\right\rangle<0, \quad \text { for a nonzero } w_{i} .
$$

This shows that $\beta_{i+1} \in \mathcal{I} \backslash\{0\}$. Since $v_{i+1}=\beta_{i+1}^{-1} w_{i}, v_{i+1}$ is a real-valued vector satisfying $v_{i+1}=\delta^{E} v_{i+1}$. Finally,

$\alpha_{i+1}=\left\langle v_{i+1}, w_{i+1}\right\rangle_{\mathrm{b}}=\left\langle M_{2}^{-1} v_{i+1}, D M_{2}^{-1} v_{i+1}\right\rangle=0$.

Note that $\left\langle M_{2}^{-1} w_{i}, w_{i}\right\rangle$ for real- or imaginary-valued vectors, $w_{i}$ can be zero if and only if $w_{i}=0$. The modified Lanczos algorithm cannot break down in the lossless case if the real-valued source vector satisfies $Q=\delta^{E} Q$

One can prove a similar result if the source vector satisfies $Q=\delta^{H} Q$, i.e., no external electric-current sources are present. The theorem shows us that in the lossless case and in case there are no external magnetic-current sources, the odd numbered Lanczos vectors built up the approximation to the electricfield strength and the even-numbered Lanczos vectors built up the approximation to the magnetic-field strength. It is also observed that in the lossless case, matrix $T_{m}$ is always diagonalizable. Further, the eigenvalues of the matrix $D M_{2}^{-1}$ are all imaginary, and the same is true for the eigenvalues of matrix $T_{m}$. In fact, in the lossless case, one can use the standard Lanczos algorithm for skew-symmetric matrices to construct the reduced-model approximation. Our modified Lanczos algorithm reduces to a variant of this standard Lanczos scheme in case of a lossless medium.

\section{APPENDIX B}

In order to show that the reduced-model approximation is a real quantity, we will use the result that the $\alpha_{i}$, as generated by the modified Lanczos algorithm, are all real and that the $\beta_{i}$ and the corresponding Lanczos vectors $v_{i}$ are either real or imaginary. We state this result as a theorem.

Theorem 3: The coefficients generated by the modified Lanczos algorithm satisfy

$$
\begin{aligned}
& \alpha_{i} \in \mathbb{R}, \quad i=1,2, \cdots \\
& \beta_{i}=\sqrt{\frac{\tau_{i-1}}{\tau_{i}}} \gamma_{i}, \gamma_{i} \in \mathbb{R}, \tau_{i}= \pm 1, \quad i=2,3, \cdots
\end{aligned}
$$

and the corresponding Lanczos vectors are of the form

$$
v_{i}=\sqrt{\tau_{i}} z_{i}, z_{i} \in \mathbb{R}^{N}, \quad i=1,2, \cdots .
$$

Proof: Let us write $\beta_{1}=\gamma_{1} / \sqrt{\tau_{1}}$, then $v_{1}=$ $\left(\sqrt{\tau_{1}} / \gamma_{1}\right) Q$. From the condition $\left\langle v_{1}, v_{1}\right\rangle_{\mathrm{b}}=1$ it follows that $\gamma_{1}^{2}=\tau_{1}\langle Q, Q\rangle_{\mathrm{b}}$. Set $\tau_{1}=\operatorname{sign}\left(\langle Q, Q\rangle_{\mathrm{b}}\right)$ then $\gamma_{1} \in \mathbb{R}$ and $v_{1}=\sqrt{\tau_{1}} z_{1}$ with $z_{1}=Q / \gamma_{1} \in \mathbb{R}^{N}$. Obviously, we have $\alpha_{1} \in \mathbb{R}$. The rest of the proof follows by induction over $i$.

The Lanczos algorithm is based on the three-term recurrence relation

$$
A v_{i}=\beta_{i+1} v_{i+1}+\alpha_{i} v_{i}+\beta_{i} v_{i-1}
$$

and for $i=1$ it follows from this relation that

$$
\beta_{2} v_{2}=\sqrt{\tau_{1}} r_{1}
$$

with $r_{1}=\left(A-\alpha_{1} E\right) z_{1} \in \mathbb{R}^{N}$. Let us write $\left.\beta_{2}=\sqrt{\left(\tau_{1} / \tau_{2}\right.}\right) \gamma_{2}$ then $v_{2}=\left(\sqrt{\tau_{2}} / \gamma_{2}\right) r_{1}$ and it follows from the condition that $\left\langle v_{2}, v_{2}\right\rangle_{\mathrm{b}}=1$ that $\gamma_{2}^{2}=\tau_{2}\left\langle r_{1}, r_{1}\right\rangle_{\mathrm{b}}$. Set $\tau_{2}=\operatorname{sign}\left(\left\langle r_{1}, r_{1}\right\rangle_{\mathrm{b}}\right)$ then $\gamma_{2} \in \mathbb{R}, v_{2}=\sqrt{\tau_{2}} z_{2}$ with $z_{2}=r_{1} / \gamma_{2} \in \mathbb{R}^{N}$ and $\alpha_{2} \in \mathbb{R}$. 
From (67) and by the induction hypothesis, we have

$$
\beta_{i+1} v_{i+1}=\sqrt{\tau_{i}} r_{i}
$$

with $r_{i}=\left(A-\alpha_{i} E\right) z_{i}-\left(\tau_{i-1} / \tau_{i}\right) \gamma_{i} z_{i-1} \in \mathbb{R}^{N}$. Let us write $\left.\beta_{i+1}=\sqrt{\left(\tau_{i} / \tau_{i+1}\right.}\right) \gamma_{i+1}$ then $v_{i+1}=\left(\sqrt{\tau_{i+1}} / \gamma_{i+1}\right) r_{i}$. From the condition that $\left\langle v_{i+1}, v_{i+1}\right\rangle_{\mathrm{b}}=1$ we have $\gamma_{i+1}^{2}=$ $\tau_{i+1}\left\langle r_{i}, r_{i}\right\rangle_{\mathrm{b}}$. Set $\tau_{i+1}=\operatorname{sign}\left(\left\langle r_{i}, r_{i}\right\rangle_{\mathrm{b}}\right)$ then $\gamma_{i+1} \in \mathbb{R}$ and $v_{i+1}=\sqrt{\tau_{i+1}} z_{i+1}$ with $z_{i+1}=r_{i} / \gamma_{i+1} \in \mathbb{R}^{N}$ and $\alpha_{i+1} \in \mathbb{R}$.

Let us define the $m \times m$ matrix $J_{m}$ as

$$
J_{m}=\operatorname{diag}\left(\sqrt{\tau_{1}}, \sqrt{\tau_{2}}, \cdots, \sqrt{\tau_{m}}\right)
$$

and let the real $N \times m$ matrix $Z_{m}$ have the column partitioning $Z_{m}=\left(z_{1}, z_{2}, \cdots, z_{m}\right)$, then

$$
V_{m}=Z_{m} J_{m}
$$

Obviously, $J_{m}^{-1}=\bar{J}_{m}$, where the overbar denotes complex conjugation. From the above theorem it follows that matrix $T_{m}$, as given in (21), can be written as

$$
T_{m}=\bar{J}_{m} H_{m} J_{m}
$$

where matrix $H_{m}$ is a real, tridiagonal, and nonsymmetric matrix given by $H_{m}=\operatorname{tridiag}\left(\tau_{i-1} \tau_{i} \gamma_{i}, \alpha_{i}, \gamma_{i+1}\right)$. Matrix $H_{m}$ is called a sign-symmetric matrix or pseudo-symmetric matrix, since $J_{m}^{2} H_{m}$ is a symmetric matrix and $J_{m}^{2}$ is a signature matrix. From (72), it follows that

$$
T_{m}^{k}=\bar{J}_{m} H_{m}^{k} J_{m}, \quad k=0,1, \cdots
$$

It is now easily proved that the reduced-model approximation is a real quantity. We will give the proof in the $s$-domain, but it can just as easily be given in the time domain. The reducedmodel approximation in the $s$-domain for $s$ real and $s>s_{0}$, with $s_{0}$ given in (25), can be written as

$$
\begin{aligned}
\hat{F}_{m}(s) & =\beta_{1} \hat{w}(s) M_{2}^{-1} V_{m} \frac{1}{s} \sum_{k=0}^{\infty}\left(-\frac{1}{s} T_{m}\right)^{k} e_{1} \\
& =\gamma_{1} \hat{w}(s) M_{2}^{-1} Z_{m} \frac{1}{s} \sum_{k=0}^{\infty}\left(-\frac{1}{s} H_{m}\right)^{k} e_{1}
\end{aligned}
$$

where we have used (71), (73), and $\beta_{1}=\gamma_{1} / \sqrt{\tau_{1}}$. This last expression for $\hat{F}_{m}(s)$ is real for $s$ real and $s>s_{0}$ and, therefore, its time-domain counterpart $F_{m}(t)$ must also be a real quantity.

\section{REFERENCES}

[1] A. Taflove, Computational Electrodynamics: The Finite-Difference Time-Domain Method. Norwood, MA: Artech House, 1995.

[2] V. L. Druskin and L. A. Knizhnerman, "Spectral approach to solving three-dimensional Maxwell's equations in the time and frequency domains," Radio Sci., vol. 29, no. 4, pp. 937-953, 1994.

[3] D. V. Widder, The Laplace Transform. Princeton, NJ: Princeton Univ. Press, 1946.

[4] P. Henrici, Applied and Computational Complex Analysis (Interscience Series 2). New York: Wiley, 1991.

[5] K. S. Yee, "Numerical solution of initial boundary value problems involving Maxwell's equations in isotropic media," IEEE Trans. Antennas Propagat., vol. AP-14, pp. 302-307, Mar. 1966.

[6] R. Lee and A. C. Cangellaris, "A study of discretization error in the finite element approximation of wave solutions," IEEE Trans. Antennas Propagat., vol. 40, pp. 542-549, May 1992.
[7] D. L. Alumbaugh, G. A. Newman, L. Prevost, and J. N. Shadid, "Threedimensional wideband electromagnetic modeling on massively parallel computers," Radio Sci., vol. 31, no. 1, pp. 1-23, 1996.

[8] G. H. Golub and C. F. Van Loan, Matrix Computations. Baltimore, MD: The Johns Hopkins Press, 1989.

[9] B. N. Parlett, The Symmetric Eigenvalue Problem. Englewood Cliffs, NJ: Prentice-Hall, 1980.

[10] R. P. Ratowsky, J. A. Fleck, Jr., and M. D. Feit, "Accurate solution of the Helmholtz equation by Lanczos orthogonalization for media with loss or gain," Opt. Lett., vol. 17, no. 1, pp. 10-12, 1992.

[11] V. L. Druskin and L. A. Knizhnerman, "Two polynomial methods of calculating functions of symmetric matrices," USSR Comput. Math. Math. Phys., vol. 29, no. 6, pp. 112-121, 1989.

[12] _ _ "Error bounds in the simple Lanczos procedure for computing functions of symmetric matrices and eigenvalues," USSR Comput. Math. Math. Phys., vol. 31, no. 7, pp. 20-30, 1991.

[13] T. Wang and A. C. Tripp, "FDTD simulation of EM wave propagation in 3-D media," Geophys., vol. 61, no. 1, pp. 110-120, 1996.

[14] Z. S. Sacks, D. M. Kingsland, R. Lee, and J. F. Lee, "A perfectly matched anisotropic absorber for use as an absorbing boundary condition," IEEE Trans. Antennas Propagat., vol. 43, pp. 1460-1463, Dec. 1995.

[15] R. W. Ziolkowski, "The design of Maxwellian absorbers for numerical boundary conditions and for practical applications using engineered artificial materials," IEEE Trans. Antennas Propagat., vol. 45, pp. 656-671, Apr. 1997.

[16] V. L. Druskin and L. A. Knizhnerman, "Krylov subspace approximation of eigenpairs and matrix functions in exact and computer arithmetic," Numer. Linear Algebra Applicat., vol. 2, no. 3, pp. 205-217, 1995.

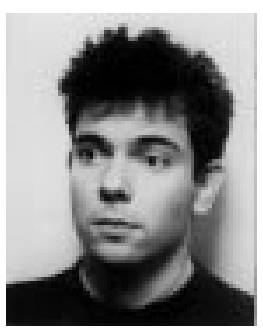

Rob F. Remis was born in Rotterdam, The Netherlands, on August 21, 1970. He received the M.Sc. degree in electrical engineering from Delft University of Technology, Delft, The Netherlands, in 1994 and is currently working toward the Ph.D. degree.

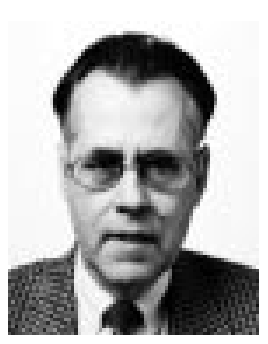

Peter M. van den Berg was born in Rotterdam, The Netherlands, on November 11, 1943. He received the degree in electrical engineering from the Polytechnical School of Rotterdam, Rotterdam, The Netherlands, in 1964, the B.Sc. and M.Sc degrees in electrical engineering, and the Ph.D. degree in technical sciences, from Delft University of Technology, Delft, The Netherlands, in 1966 , 1968, and 1971, respectively.

From 1967 to 1968, he was employed as a Research Engineer by the Dutch Patent Office. Since 1968, he has been a Scientific Staff Member of the Electromagnetic Research Group, Delft University of Technology, during which time he has carried out research and taught classes in the area of wave propagation and scattering problems, and in 1981, he became a Professor. From 1973 to 1974, he was Visiting Lecturer in the Department of Mathematics, University of Dundee, Scotland. During a three-month period from 1980 to 1981, he was a Visiting Scientist at the Institute of Theoretical Physics, Goteborg, Sweden. From 1988 to 1994, he also carried out research at the Center of Mathematics of Waves, University of Delaware. During the summers of 1993 to 1995 , he was a Visiting Scientist at Schell Research B.V., Rijswijk, The Netherlands. Since 1994, he has also been a Professor in the Delft Research School Centre of Technical Geoscience. At present, his main research interest is the efficient computation of field problems using iterative techniques based on error minimization, the computation of fields in strongly inhomogeneous media, and the use of wave phenomena in seismic data processing. His major interest is in an efficient solution of the nonlinear inverse scattering problem.

Prof. van den Berg was the recipient of an award from Niels Stensen Stichting, The Netherlands (1973-1974), and was the recipient of a NATO award. 